\title{
The Influence of the Model Fractal Parameters on the Electromagnetic Response in Environment with Cylindrical Layers
}

\author{
Valcir João da Cunha Farias ${ }^{1}$, Brígida Ramati Pereira da Rocha ${ }^{2}$, Alice Nabiça Mores ${ }^{1}$ \\ ${ }^{1}$ Faculty of Statistics, Federal University of Pará, Belém, Brazil \\ ${ }^{2}$ Pos-Graduate Program in Electrical Engineering, Federal University of Pará, Belém, Brazil \\ Email:valcir@ufpa.br
}

How to cite this paper: Farias, V.J.C., da Rocha, B.R.P. and Mores, A.N. (2017) The Influence of the Model Fractal Parameters on the Electromagnetic Response in Environment with Cylindrical Layers. International Journal of Geosciences, 8, 349-363. https://doi.org/10.4236/ijg.2017.83018

Received: December 16, 2016

Accepted: March 28, 2017

Published: March 31, 2017

Copyright $\odot 2017$ by authors and Scientific Research Publishing Inc. This work is licensed under the Creative Commons Attribution International License (CC BY 4.0).

http://creativecommons.org/licenses/by/4.0/

\begin{abstract}
The induced polarization response of an environment presenting cylindrical layers was obtained. The fractal model for complex resistivity was employed as an intrinsic property of the polarizable layers. The influence of the model fractal parameters on the electromagnetic response was investigated. The results demonstrated that the fractal parameters dominate the apparent resistivity phase response; measurements of the induced polarization data allow for the determination of the fractal properties of the environment without noticeable electromagnetic coupling effects at frequencies below $10^{4} \mathrm{~Hz}$.
\end{abstract}

\section{Keywords}

Fractal Model, Induced Polarization, Complex Resistivity, Well Log

\section{Introduction}

The induced polarization effect has an electrochemical origin and is usually associated with geological and biological environments [1] [2] [3]. As a consequence of this effect, electrical resistivity values in these environments are complex and frequency-dependent.

In geophysics, the induced polarization method uses fact that the constituent parameters of rocks (conductivity and permittivity) are frequency-dependent to carry out prospecting activities. This method was originally applied to the prospecting of disseminated ores, and has gradually evolved over the years, currently being used in mineral discrimination [4] and environmental studies [5] and [6].

The quantitative interpretation of field induced polarization data is a difficult task due to the fractal nature of geological environments and the inductive 
coupling caused by electromagnetic interactions between the environment and the electrode arrays used for current injection and potential measurements. The interpretation of these kinds of data requires a physical model to explain the behavior of a polarizable environment in an ample frequency range.

Several relaxation models have been proposed to describe the electrical polarization of rocks, in the works of Debye [7], Cole-Cole [8], Davidson and Cole [9] and Dias [10], each taking into account a certain specific characteristic for a given frequency range, limited to $10^{2} \mathrm{~Hz}$. Relaxation models demonstrate the general behavior of the amplitude spectrum and the complex resistivity phase (conductivity) at different frequencies for different types of materials. The most widely used model is the Cole-Cole model, which does not, however, consider the fractal nature of the environment.

Rocha [11] developed a model that considers the fractal effects of porous surfaces and includes rock volume response, namely the fractal model for complex resistivity. This model accounts for the electrical properties of rocks at a higher frequency range than traditional models. The introduction of the roughness factor in this model allows for the investigation of rock texture, which is very important when attempting to describe the electrical behavior of rocks. This means that parameters representing the fractal geometry of the environment exist, which may be, in turn, related to rock texture. With this, it is possible to obtain important and accurate geological information of the subsurface from electrical data obtained on the terrain surface.

Rocha [11] and Rocha \& Habshy [3] determined the response of a terrain presenting three horizontal layers, with the second layer polarizable with its intrinsic properties given by the fractal model for complex resistivity of [11] and analyzed the induced polarization response. These authors observed that the parameters related to the fractal geometry of the model dominate the phase response of the apparent complex resistivity at low frequencies, and also found that the fractal exponent does not depend on the electrical properties of the fluid filling the rock cavities.

The fact that the fractal exponent is independent from the electrical resistivity of the percolating solutions avoids any influence of the invasion zone in the electric profiling of wells. Thus, it is interesting to investigate the response of a polarizable medium in well environments by applying the fractal model for complex resistivity.

Farias et al. [12] [13] simulated the fractal model for complex resistivity as being an intrinsic electrical property of horizontal environments with superficial and volumetric formations (2-D and 3-D geological models), with applications for both contaminated and non-contaminated environments. The results demonstrated that anomalies are well-detected and observable by images of the parameter distribution of the fractal model, being an alternative in the detection of anomalies in the geologic environment, such as in the study of environmental contamination. The fractal complex resistivity model, however, has not yet been applied as an intrinsic electrical property in the analysis of the polarization re- 
sponse of cylindrical environments.

The main aim of electrical well profiling is to estimate the electrical resistivity of the geological formation where the well is inserted. However, the response of the resistivity profiling is influenced by the resistivity of the formation itself, as well as by the invaded zone, which is generated during the drilling process. Therefore, the effects of this invaded area should be avoided.

In the present study, the fractal model for complex resistivity [11] is employed as an intrinsic electrical property of an environment with cylindrical layers (the well, invaded zone and formation) to evaluate the influence of the parameters of the fractal model in the induced polarization response in this geological geometry. The model parameters represent the fractal geometry of the environment which, as presented previously, can be related to the texture of the rocks in the analyzed environment.

\section{The Fractal Model}

Representing the time dependence of the electric field as $e^{-i \omega t}$, the expression proposed by [4] for the complex resistivity $\rho(\omega)$ is:

$$
\rho(\omega)=\rho_{o}\left[1-m\left(1-\frac{1}{1+\frac{1+u}{\delta_{r}(1+v)}}\right)\right] \gamma_{h}
$$

where $\rho_{o}$ is the DC resistivity of the material; $m$ is the chargeability defined by [14]; $\delta_{r}$ is the parameter that relates the resistivity of the conductive grains blocking the pores of the geologic environment to the DC resistivity of the rock matrix; $\quad \gamma_{h}=1 /\left(1+i \omega \tau_{o}\right) ; \quad u=i \omega \tau(1+v) ; \quad v=\left(i \omega \tau_{f}\right)^{-\eta} ; \tau$ is the relaxation time constant related to the double-layer oscillations; $\tau_{o}$ is the relaxation time constant associated with the material as a whole; $\tau_{f}$ is the time of fractal relaxation, related to the time involved in the charge and energy transfer in the rough interfaces; and $\eta$ is the parameter directly related to the fractal geometry of the environment, determined by the type and distribution of the mineral that causes the polarization at low frequencies.

Some typical values of the fractal model parameters for complex resistivity cited by [11], are: $\rho_{o}=10^{2} \Omega \cdot \mathrm{m} ; m=0,5 ; \delta_{r}=1 ; \eta=0,5 ; \tau=10^{-6} \mathrm{~s}$; $\tau_{f}=10^{-3} \mathrm{~s}$ and $\tau_{o}=10^{-12} \mathrm{~s}$.

\section{Induced Polarization Response in a Stratified Cylindrical Environment}

Normally a four-electrode configuration is used to measure the complex resistivity of a geological environment. An electric current is introduced into the environment via an electrode pair $(A$ and $B$ ) and the voltage is measured by the other electrode pair ( $M$ and $N$ ). Figure 1 illustrates the four-electrode configuration used in the present study to determine the apparent resistivity on an environment presenting cylindrical layers. 


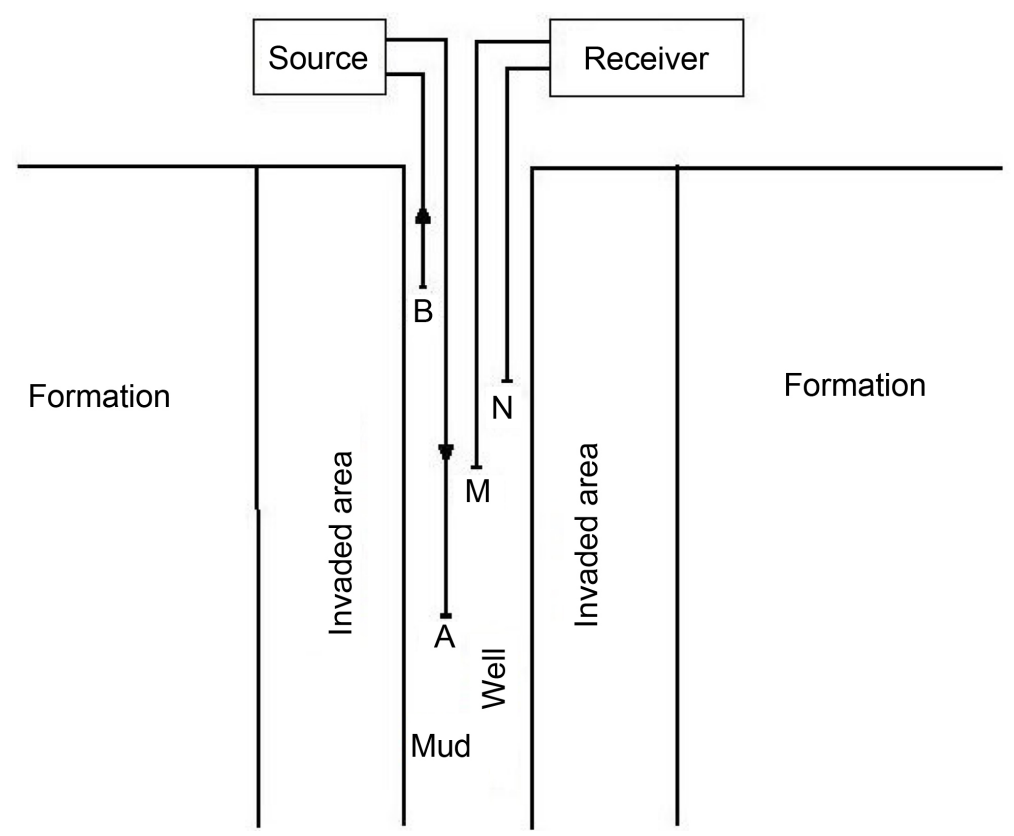

Figure 1. Illustration of the four-electrode configuration applied to a stratified cylindrical environment.

To calculate the potential measured by the receiver electrode pair, the electromagnetic problem for a four-electrode configuration must be solved. From Maxwell equations and assuming a time dependence of the $e^{-i \omega t}$ type, we have:

$$
\begin{gathered}
\nabla \times \bar{E}=i \omega \mu \bar{H} \\
\nabla \times \bar{H}=-i \omega \varepsilon \bar{E}+\sigma \bar{E}+\bar{J}_{S}
\end{gathered}
$$

where $\bar{J}_{S}$ is the current density due to the source; $\varepsilon$ is the effective dielectric constant and $\mu$ is the permeability of the environment and is approximated by the permeability of vacuum. Combining the conductivity $(\sigma)$ and displacement factor $(i \omega \varepsilon)$, the current density can be denoted as:

$$
\bar{J}=\sigma * \bar{E}
$$

and (3) can be denoted as:

$$
\nabla \times \bar{H}=\bar{J}+\bar{J}_{S}
$$

When applying the divergence operator to (2) and (5), the following equations are obtained:

$$
\begin{gathered}
\nabla \cdot \bar{H}=0 \\
\nabla \cdot \bar{J}=-\nabla \cdot \bar{J}_{S}
\end{gathered}
$$

where the left side of (7) is a result of the charge accumulation caused by the injected current. When observing (6), the Maxwell equations can be displayed in terms of a vector potential $A$ and scalar potential $\phi$

$$
\begin{gathered}
\bar{H}=\nabla \times \bar{A} \\
\bar{E}=i \omega \mu_{\circ} \bar{A}-\nabla \phi
\end{gathered}
$$

observing (4), (5), (8) and (9), and considering the condition 


$$
\nabla \cdot \bar{A}=-\sigma * \phi
$$

where $\sigma^{*}=\sigma-i \omega \varepsilon$ is the complex conductivity. Thus, the wave equations below are obtained:

$$
\begin{gathered}
\nabla^{2} \bar{A}+k^{2} \bar{A}=-\bar{J}_{S} \\
\nabla^{2} \phi+k^{2} \phi=\frac{1}{\sigma^{*}} \nabla \cdot \bar{J}_{S}
\end{gathered}
$$

with $k^{2}=i \omega \mu \sigma^{*}$. Specifying the current density in cylindrical coordinates, with

$$
\bar{J}_{S}=-\frac{I \delta(r)\left[u\left(z-z_{1}\right)-u\left(z-z_{2}\right)\right]}{r} e_{z}
$$

where $u$ is a Heaviside function, $I$ is the current intensity, $z_{1}$ and $z_{2}$ are the positions of the current electrodes $(A e B)$ and $e_{z}$ is the unit vector in the $z$ direction.

Considering (8), (9) and (10) and the fact that the vector potential is of the $\left(0,0, A_{z}\right)$ form, the following equations are obtained:

$$
\begin{gathered}
E_{z}=i \omega \mu_{o} A_{z}+\frac{1}{\sigma^{*}} \frac{\partial^{2} A_{z}}{\partial z^{2}} \\
H_{\theta}=\frac{\partial A_{z}}{\partial \rho}
\end{gathered}
$$

With $A_{1 z}$ being the solution for (11) in the internal region of the well, then

$$
\nabla^{2} \bar{A}_{1 z}+k_{1}^{2} \bar{A}_{1 z}=-\frac{I \delta(r)\left[u\left(z-z_{1}\right)-u\left(z-z_{2}\right)\right]}{r}
$$

is valid for $r \leq a$, where $a$ is the well radius. If $A_{2 z}$ is the solution to (11) in the invaded zone, then

$$
\nabla^{2} \bar{A}_{2 z}+k_{2}^{2} \bar{A}_{2 z}=0
$$

is valid for $a \leq r \leq b$, where $b$ is the radius of the invaded zone. In the same manner, regarding the formation, with a solution $A_{3 z}$, the following equation is obtained:

$$
\nabla^{2} \bar{A}_{3 z}+k_{3}^{2} \bar{A}_{3 z}=0
$$

for $r \geq b$. The tangential components of $\bar{E}$ and $\bar{H}$ are continuous in the interfaces, or

$$
E_{1 z}=E_{2 z} \text { and } H_{1 \theta}=H_{2 \theta} \quad \text { in } \quad r=a
$$

and

$$
E_{2 z}=E_{3 z} \text { and } H_{2 \theta}=H_{3 \theta} \text { in } r=b
$$

thus, by using (14) and (15) the boundary conditions to solve (16), (17) and (18) are obtained.

$$
\begin{gathered}
\left(i w \mu_{o} A_{1 z}+\frac{1}{\sigma^{*}} \frac{\partial^{2} A_{1 z}}{\partial z^{2}}\right)_{r=a}=\left(i w \mu_{o} A_{2 z}+\frac{1}{\sigma^{*}} \frac{\partial^{2} A_{2 z}}{\partial z^{2}}\right)_{r=a} \\
\left(\frac{\partial A_{1 z}}{\partial r}\right)_{r=a}=\left(\frac{\partial A_{2 z}}{\partial r}\right)_{r=a}
\end{gathered}
$$




$$
\begin{gathered}
\left(i w \mu_{0} A_{21 z}+\frac{1}{\sigma^{*}} \frac{\partial^{2} A_{21 z}}{\partial z^{2}}\right)_{r=b}=\left(i w \mu_{0} A_{3 z}+\frac{1}{\sigma^{*}} \frac{\partial^{2} A_{3 z}}{\partial z^{2}}\right)_{r=b} \\
\left(\frac{\partial A_{2 z}}{\partial r}\right)_{r=b}=\left(\frac{\partial A_{3 z}}{\partial r}\right)_{r=b}
\end{gathered}
$$

In order to solve Equations (16), (17) and (18) subject to the boundary Equations (19), (20), (21) and (22), it is convenient to introduce the Green $G\left(r, z-z^{\prime}\right)$ equation, that satisfies the following equations:

$$
\begin{gathered}
\nabla^{2} G_{1 z}+u_{1}^{2} G_{1 z}=\frac{I \delta(r) \delta\left(z-z^{\prime}\right)}{r} \text { for } r \leq a \\
\nabla^{2} G_{2 z}+u_{2}^{2} G_{2 z}=0 \text { for } a \leq r \leq b \\
\nabla^{2} G_{3 z}+u_{3}^{2} G_{3 z}=0 \text { for } r \geq b
\end{gathered}
$$

subject to the boundary conditions below:

$$
\begin{gathered}
\left(i w \mu_{o} G_{1 z}+\frac{1}{\sigma^{*}} \frac{\partial^{2} G_{1 z}}{\partial z^{2}}\right)_{r=a}=\left(i w \mu_{o} G_{2 z}+\frac{1}{\sigma^{*}} \frac{\partial^{2} G_{2 z}}{\partial z^{2}}\right)_{r=a} \\
\left(\frac{\partial G_{1 z}}{\partial r}\right)_{r=a}=\left(\frac{\partial G_{2 z}}{\partial r}\right)_{r=a} \\
\left(i w \mu_{o} G_{21 z}+\frac{1}{\sigma^{*}} \frac{\partial^{2} G_{21 z}}{\partial z^{2}}\right)_{r=b}=\left(i w \mu_{o} G_{3 z}+\frac{1}{\sigma^{*}} \frac{\partial^{2} G_{3 z}}{\partial z^{2}}\right)_{r=b} \\
\left(\frac{\partial G_{2 z}}{\partial r}\right)_{r=b}=\left(\frac{\partial G_{3 z}}{\partial r}\right)_{r=b}
\end{gathered}
$$

The solutions to Equations (23), (24) and (25) are given by:

$$
G_{1 z}=\frac{-I e^{i u_{1} R}}{4 \pi R}+\int_{0}^{\infty}\left[A\left(k_{z}\right) K_{o}\left(k_{1 r} r\right)+B\left(k_{z}\right) I_{o}\left(k_{1 r} r\right)\right] \cos k_{z}\left(z-z^{\prime}\right) \mathrm{d} k_{z}
$$

Since $G_{1 z}$ is regular in $r=0$, then $A\left(k_{z}\right)=0$, thus:

$$
G_{1 z}=\frac{-I e^{i u_{1} R}}{4 \pi R}+\int_{0}^{\infty} B\left(k_{z}\right) I_{o}\left(k_{1 r} r\right) \cos k_{z}\left(z-z^{\prime}\right) \mathrm{d} k_{z}
$$

Using the identity (15) [15]:

$$
\frac{e^{i u_{1} R}}{R}=\frac{2}{\pi} \int_{0}^{\infty} K_{o}\left(k_{1 r} r\right) \cos k_{z}\left(z-z^{\prime}\right) \mathrm{d} k_{z}
$$

in Equation (31), the following equation is obtained:

$$
G_{1 z}=\frac{-I}{2 \pi^{2}} \int_{0}^{\infty} K_{o}\left(k_{1 r} r\right) \cos k_{z}\left(z-z^{\prime}\right) \mathrm{d} k_{z}+\int_{0}^{\infty} B\left(k_{z}\right) I_{o}\left(k_{r} r\right) \cos k_{z}\left(z-z^{\prime}\right) \mathrm{d} k_{z}
$$

valid for $r \leq a$;

$$
G_{2 z}=\int_{0}^{\infty}\left[C\left(k_{z}\right) K_{o}\left(k_{2 r} r\right)+D\left(k_{z}\right) I_{o}\left(k_{2 r} r\right)\right] \cos k_{z}\left(z-z^{\prime}\right) \mathrm{d} k_{z}
$$

valid for $a \leq r \leq b$; and

$$
G_{3 z}=\int_{0}^{\infty} E\left(k_{z}\right) K_{o}\left(k_{3 r} r\right) \cos k_{z}\left(z-z^{\prime}\right) \mathrm{d} k_{z}
$$

in $r \geq b$, where $I_{o}$ and $K_{o}$ are modified Bessel functions of order zero of the first and second species, respectively, and $k_{j r}(j=1,2,3)$ and $R$ are given by: 


$$
k_{j r}=\sqrt{k_{z}^{2}-u_{j}^{2}} \quad R=\sqrt{r^{2}-\left(z-z^{\prime}\right)^{2}} .
$$

The vector potential function $A_{j}(r, z)$ is determined by the integral of the Green function $\left(G_{l}\right)$ :

$$
A_{l}(r, z)=\int_{z_{2}}^{z_{1}} G_{j}\left(r, z-z^{\prime}\right) \mathrm{d} z^{\prime}
$$

Imposing boundary conditions (26)-(29) in Equations (32), (33) and (34), functions $B, C, D$ and $E$ are determined. In this way, the following system of equation is obtained:

$$
\begin{aligned}
& k_{1 r}^{2} I_{o}\left(k_{1 r} a\right) B-\frac{\sigma_{1}^{*}}{\sigma_{2}^{*}} k_{2 r}^{2}\left[K_{o}\left(k_{2 r} a\right) C+I_{o}\left(k_{2 r} a\right) D\right]=\frac{I k_{1 r}^{2}}{2 \pi^{2}} K_{o}\left(k_{1 r} a\right) ; \\
& k_{1 r} I_{1}\left(k_{1 r} a\right) B+k_{2 r}\left[K_{1}\left(k_{2 r} a\right) C-I_{1}\left(k_{2 r} a\right) D\right]=\frac{-I}{2 \pi^{2}} k_{1 r} K_{1}\left(k_{1 r} a\right) ; \\
& k_{2 r}^{2}\left[K_{o}\left(k_{2 r} b\right) C+I_{o}\left(k_{2 r} b\right) D\right]-\frac{\sigma_{2}^{*}}{\sigma_{3}^{*}} k_{3 r}^{2} K_{o}\left(k_{3 r} b\right) E=0 ; \\
& e \\
& k_{2 r}\left[K_{1}\left(k_{2 r} b\right) C-I_{1}\left(k_{2 r} b\right) D\right]-k_{3 r} K_{1}\left(k_{3 r} b\right) E=0
\end{aligned}
$$

to calculate the induced potential difference measured by electrodes $M$ and $N$ (Figure 1). Thus only the vector potential $A_{1}(r, z)$ must be obtained, which is associated with the function $B\left(k_{z}\right)$. Solving the above system, the following equations are obtained:

$$
\begin{gathered}
B\left(k_{z}\right)=\frac{\frac{I}{2 \pi^{2}}\left(F_{1} H_{1}+F_{2} H_{2}\right)}{F_{3} H_{1}+F_{4} H_{2}} \\
F_{1}=K_{o}\left(k_{1 r} a\right) K_{1}\left(k_{2 r} a\right)-\frac{\sigma_{1}^{*}}{\sigma_{2}^{*}} \frac{k_{2 r}}{k_{1 r}} K_{1}\left(k_{1 r} a\right) K_{o}\left(k_{2 r} a\right) \\
F_{2}=K_{o}\left(k_{1 r} a\right) I_{1}\left(k_{2 r} a\right)+\frac{\sigma_{1}^{*}}{\sigma_{2}^{*}} \frac{k_{2 r}}{k_{1 r}} K_{1}\left(k_{1 r} a\right) I_{o}\left(k_{2 r} a\right) \\
F_{3}=I_{o}\left(k_{1 r} a\right) K_{1}\left(k_{2 r} a\right)+\frac{\sigma_{1}^{*}}{\sigma_{2}^{*}} \frac{k_{2 r}}{k_{1 r}} I_{1}\left(k_{1 r} a\right) K_{o}\left(k_{2 r} a\right) \\
F_{4}=I_{o}\left(k_{1 r} a\right) I_{1}\left(k_{2 r} a\right)-\frac{\sigma_{1}^{*}}{\sigma_{2}^{*}} \frac{k_{2 r}}{k_{1 r}} I_{1}\left(k_{1 r} a\right) I_{o}\left(k_{2 r} a\right) \\
H_{1}=K_{1}\left(k_{3 r} b\right) I_{o}\left(k_{2 r} b\right)+\frac{\sigma_{2}^{*}}{\sigma_{3}^{*}} \frac{k_{3 r}}{k_{2 r}} K_{o}\left(k_{3 r} b\right) I_{1}\left(k_{2 r} b\right) \\
H_{2}=K_{o}\left(k_{2 r} b\right) K_{1}\left(k_{3 r} b\right)-\frac{\sigma_{2}^{*}}{\sigma_{3}^{*}} \frac{k_{3 r}}{k_{2 r}} K_{1}\left(k_{2 r} b\right) K_{o}\left(k_{3 r} b\right)
\end{gathered}
$$

When combining Equations (32) and (35), $A_{1}(r, z)$ can be written as:

$$
A_{1}(r, z)=\int_{0}^{\infty}\left[\frac{-I}{2 \pi^{2}} K_{o}\left(k_{1 r} r\right)+B\left(k_{z}\right) I_{o}\left(k_{r} r\right)\right]\left[\operatorname{sen} k_{z}\left(z-z_{2}\right)-\operatorname{sen}\left(z-z_{1}\right)\right] \frac{d k_{z}}{k_{z}}
$$

With $B\left(k_{z}\right)$ given by Equation (36). The potential difference measured by electrodes $M$ and $N$ is given by the line integral:

$$
V(r, z)=\int E_{1}(r, z) \mathrm{d} l
$$


with $\mathrm{d} l=\mathrm{dze} e_{z}$, so

$$
V(r, z)=\int E_{1}(r, z) \mathrm{d} z=\int\left(\frac{1}{\sigma_{1}^{*}} \frac{\partial^{2} A_{1}}{\partial z^{2}}+i w \mu_{o} A_{1}\right)
$$

thus,

$$
V(r, z)=\frac{1}{\sigma_{1}^{*}} \int_{0}^{\infty} \frac{k_{1 r}^{2}}{k_{z}^{2}}\left[\frac{I}{2 \pi^{2}} K_{o}\left(k_{1 r} r\right)-B\left(k_{z}\right) I_{o}\left(k_{1 r} r\right)\right]\left[\cos \left(z-z_{1}\right)-\cos \left(z-z_{2}\right)\right] \mathrm{d} k_{z}
$$

The potential $V_{M N}$ is calculated as follows:

$$
V_{M N}=V\left(r_{o}, z_{N}\right)-V\left(r_{o}, z_{M}\right)
$$

where $r_{o}$ is the radial position of the electrodes in the well and $z_{M}$ and $z_{N}$ are the vertical positions of the potential electrodes. The integral of Equation (38) is solved by quadrature technique [16] or by digital filters [17].

A similar result was found by [18] in the study of the anisotropy effect on resistivity measurements in wells and by [19] in the study of a dynamic model for resistivity and induced polarization data in wells.

\section{Results}

The induced polarization responses of the two geological two situations were obtained by applying Equation (38): 1) the environment presenting two cylindrical layers (the well and the formation); 2) the environment presenting three cylindrical layers (the well, the invaded zone and the formation). The resistivity of the mud for the two geometries was of $1 \Omega \cdot \mathrm{m}$ when disregarding the polarization effect. The distances between the electrodes, in meters, were of $0.41,6.1$, 20.9 and 26.59 for $A M, N A, B N$ and $B M$, respectively. The default value for the well radius was of $10 \mathrm{~cm}$.

In order to analyze the influence of parameters $\eta, m, \delta_{r}, \tau$ and $\tau_{f}$ of the fractal model, the simulations were carried out for three different values for each of these parameters, and when variations in a certain parameter occurred the others assumed the typical values described above.

\subsection{Environment Presenting Two Layers}

Figure 2 displays the induced polarization response for a well with only two cylindrical layers (mud and formation). The following values were used: $0.25,0.5$ and 0.75 for the fractal exponent $\eta ; 0.25,0.5$ and 0.75 for chargeability; $0.1,1$ and 10 for parameter $\delta_{i} ; 10^{-9} \mathrm{~s}, 10^{-6} \mathrm{~s}$ and $10^{-3} \mathrm{~s} 3$ for the time constant $\tau$ and $10^{-4} \mathrm{~s}$, $10^{-3} \mathrm{~s}$ and $10^{-2}$ for the fractal time constant $\tau_{f}$

It is observed from Figure 2 that the fractal model for complex resistivity can be used in a wide frequency range at environment with cylindrical layers. As in the case of horizontal layers [11], the fractal parameters $\eta, \delta_{r}$ e $\tau_{f}$ particularly the fractal exponent $\eta$ dominates the phase angle response of the apparent complex resistivity, mainly at low frequency. According to [2] [11], this feature is very important because at low frequency the parameters carry information about the roughness of the pores of rocks. Thus, it becomes possible to investigate, from 

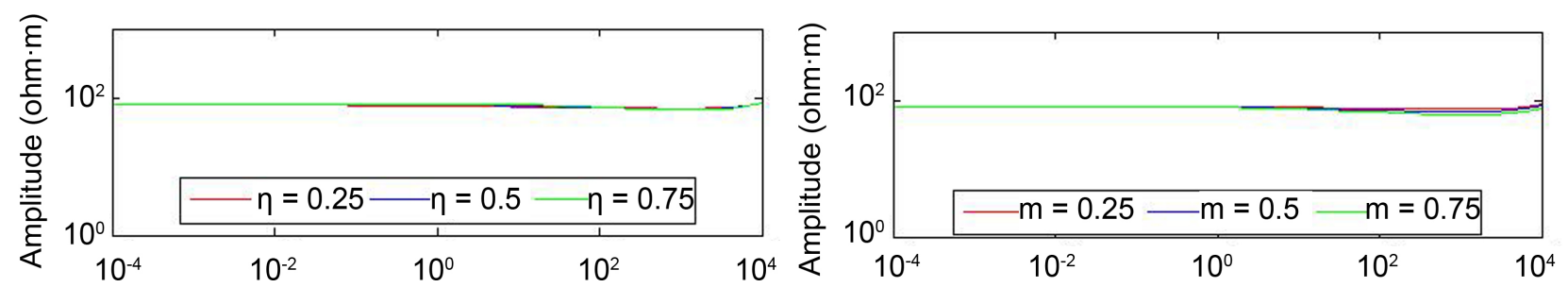

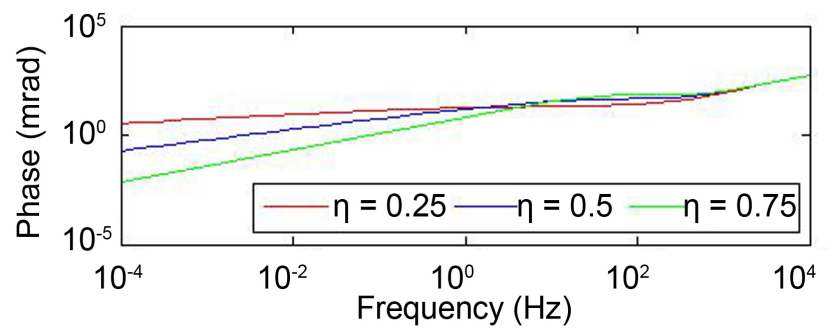

(a)
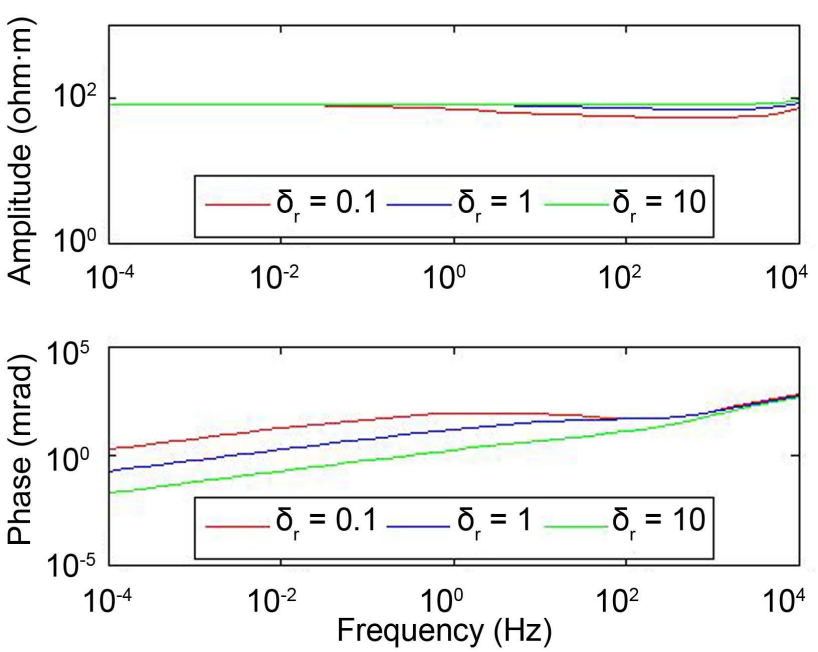

(c)

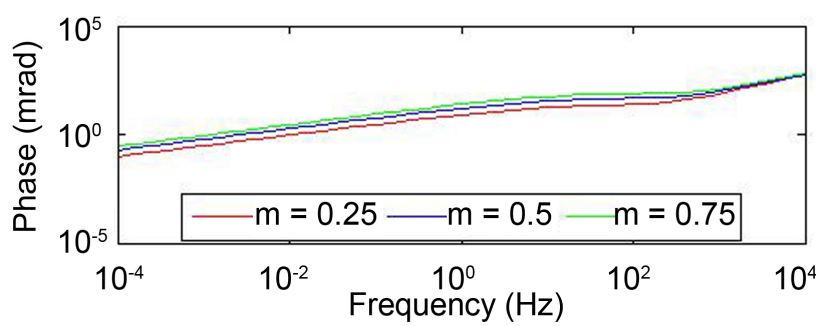

(b)
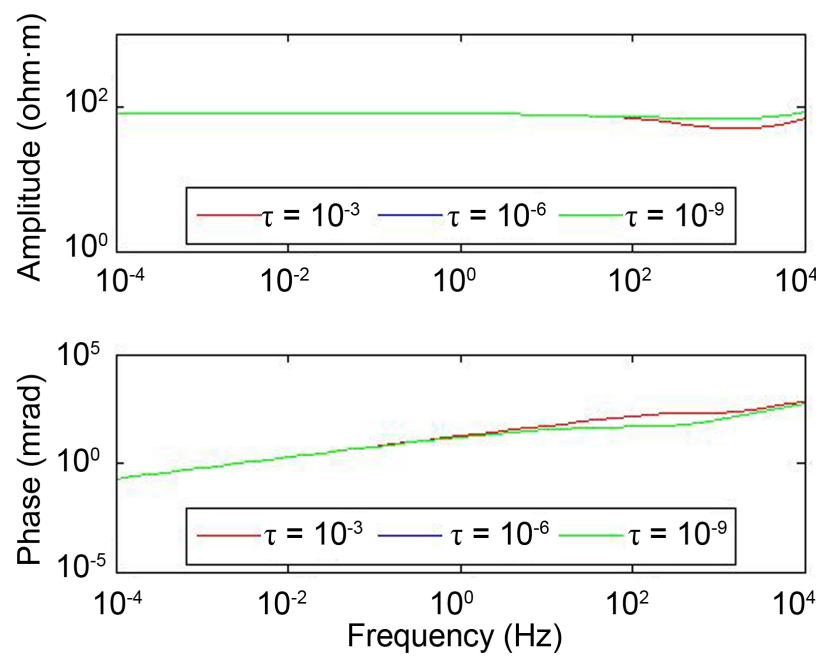

(d)
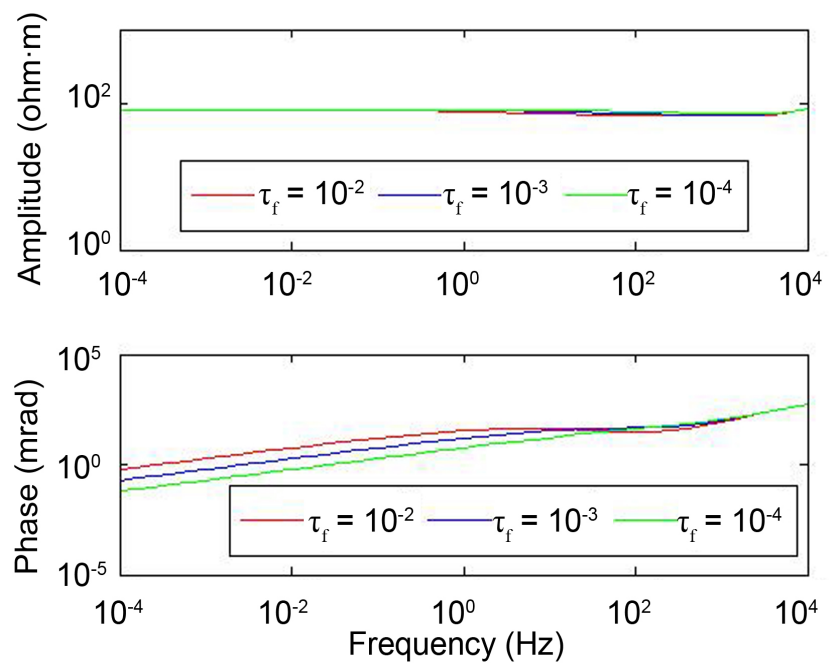

(e)

Figure 2. Amplitude and phase angle of the apparent resistivity in a well presenting mud and non-invasion of the formation. The formation is polarizable with the intrinsic electrical properties given by the fractal model when (a) varying $\eta$; (b) varying $m$; (c) varying $\delta_{r}$; $(\mathrm{d})$ varying $\tau$ and (e) varying $\tau_{f}$ 
data of induced polarization in the frequency domain in the well, the transport properties of the geological environment.

\subsection{Environment Presenting Three Layers}

Three thicknesses of the invaded zone were considered when analyzing the induced polarization response in an environment with three cylindrical layers (mud, invaded zone and formation): one, two and five times the radius of the well. The DC resistivity of the invaded area was presumed equal to $10 \Omega \cdot \mathrm{m}$. Figures 3-7 shows the response of the induced polarization when varying $\eta, m, \delta_{r}, \tau$ and $\tau_{f}$ respectively:

The amplitude response of the apparent complex resistivity was affected by the variation of the invaded zone. However, the phase angle response was only slightly affected. This is similar to the results observed by [2] [3], which demon-
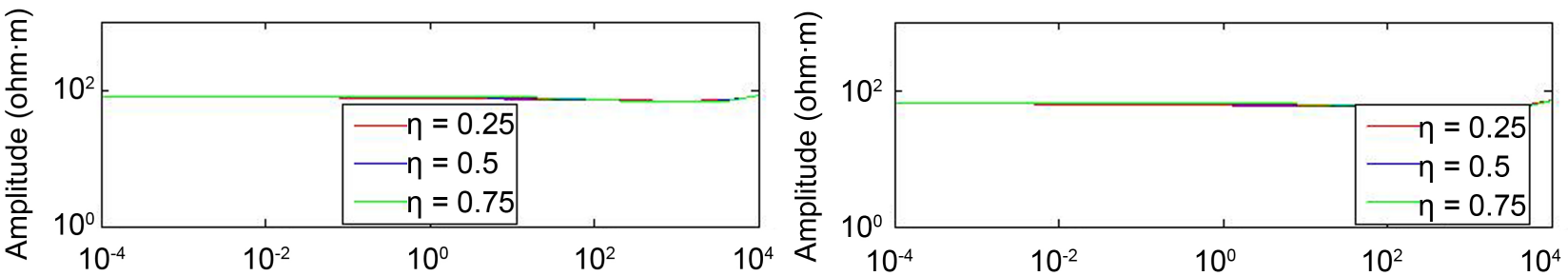

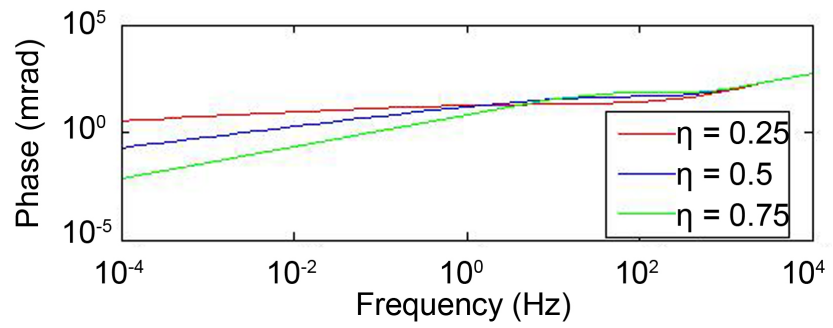

(a)

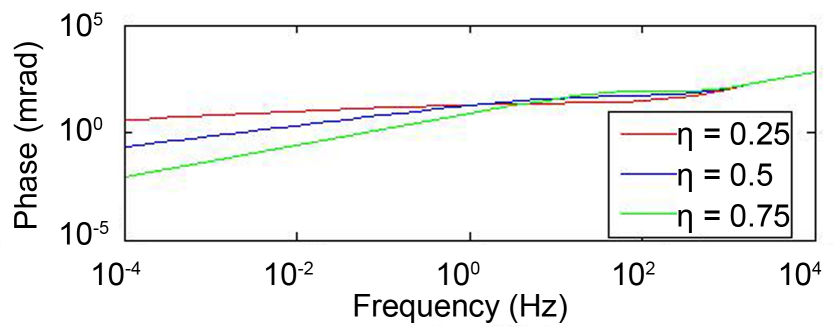

(b)
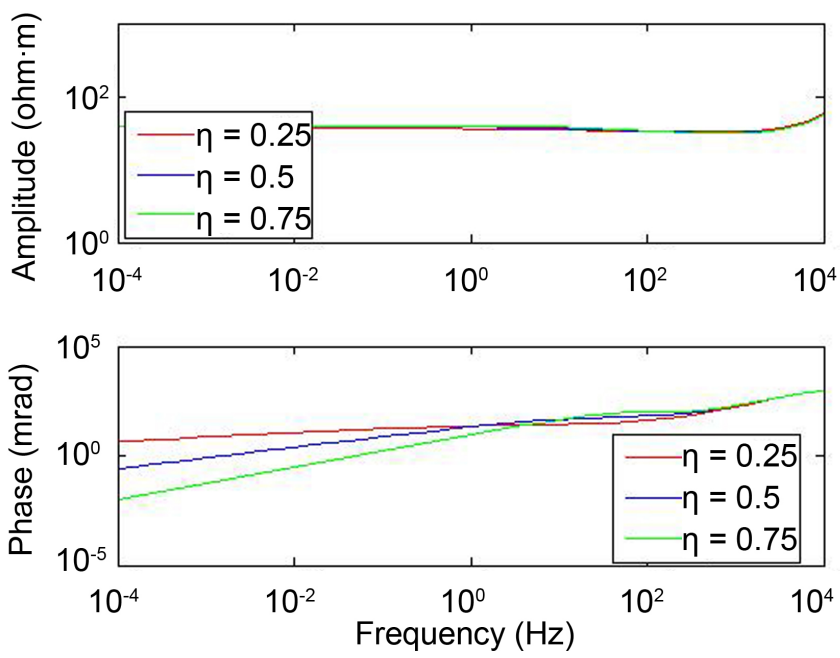

(c)

Figure 3. Amplitude and phase angle of the complex apparent resistivity in a well presenting mud, an invaded zone and formation. The invaded zone and formation are polarizable, and the intrinsic electrical properties are given by the fractal model when varying the parameter $\eta$. The radii of the invaded zone were (a) the same; (b) twice and (c) five times the well radius. 

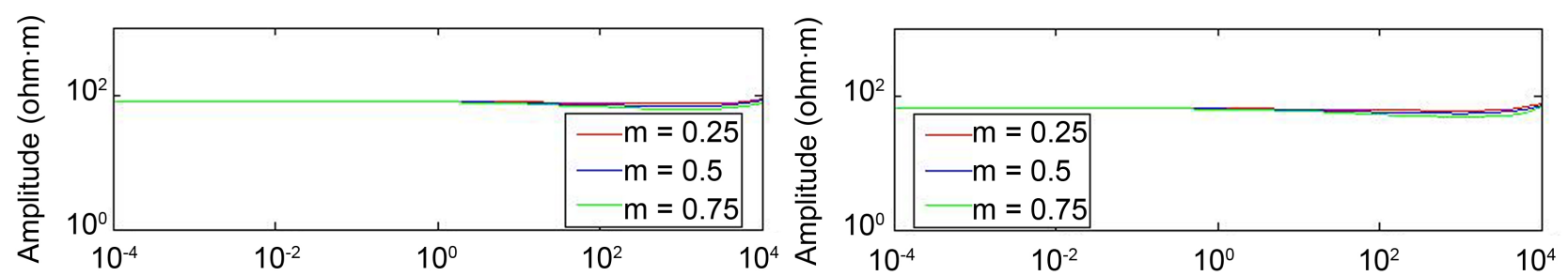

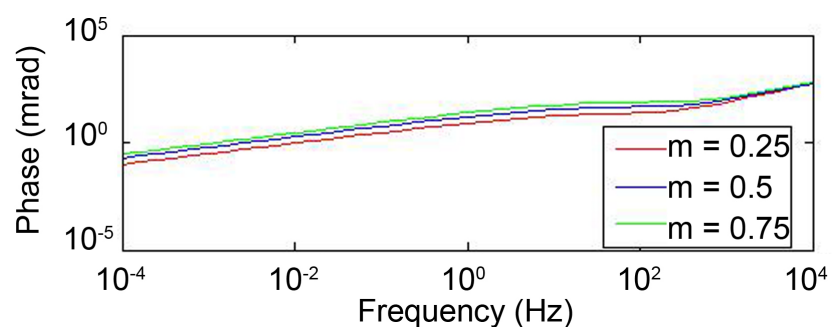

(a)

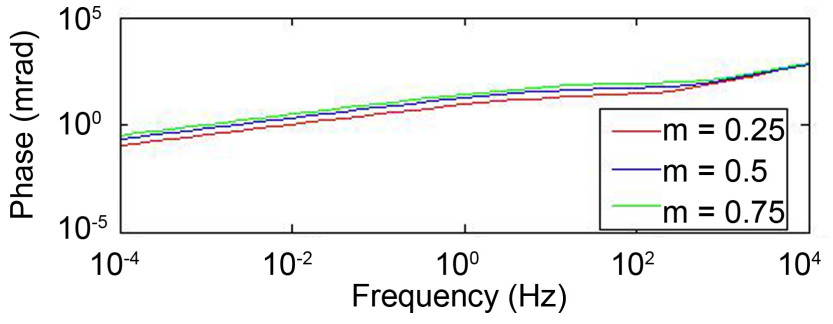

(b)
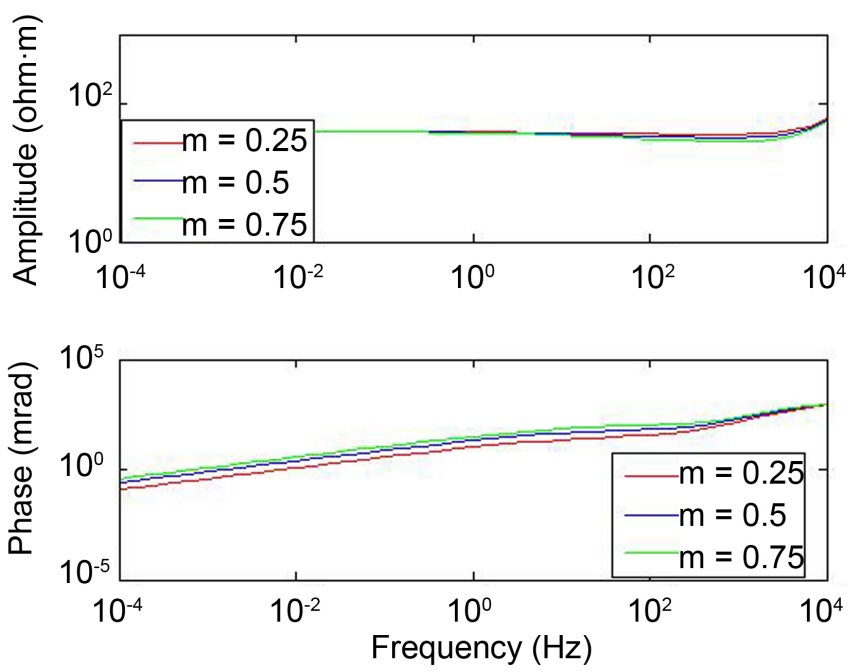

(c)

Figure 4. Amplitude and phase angle of the complex apparent resistivity in a well presenting mud, an invaded zone and formation. The invaded zone and formation are polarizable, and the intrinsic electrical properties are given by the fractal model when varying the parameter $m$ (chargeability). The radii of the invaded zone were (a) the same; (b) twice and (c) five times the well radius.

strates the fractal nature of the complex resistivity, since the scale variation in the measurements did not change the phase angle response of the cylindrical environment. In addition, the fractal exponent parameter $\eta$, which dominates the response phase, is not dependent on the electrical properties of the fluids filling the empty spaces of the rocks present in the environment, depending only on their mineralogical composition. Thus, the influence of the invaded zone is attenuated in the phase response.

\section{Conclusion}

The induced polarization response of a cylindrical stratified environment was obtained and the fractal model for complex resistivity was applied as an intrinsic electrical property of a polarizable environment presenting cylindrical layers. The influence of the model parameters on the induced polarization response was 

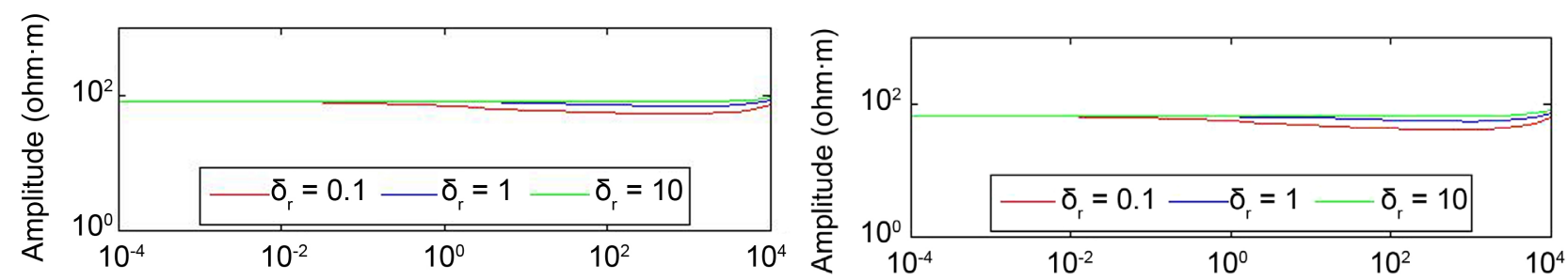

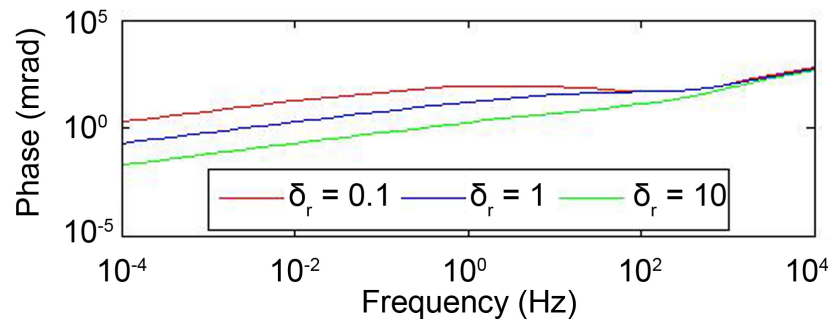

(a)

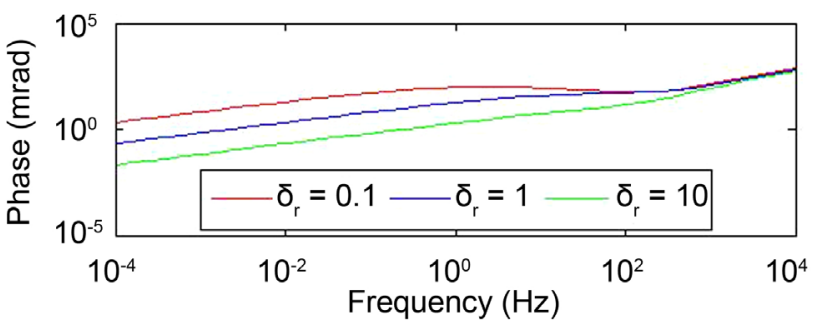

(b)
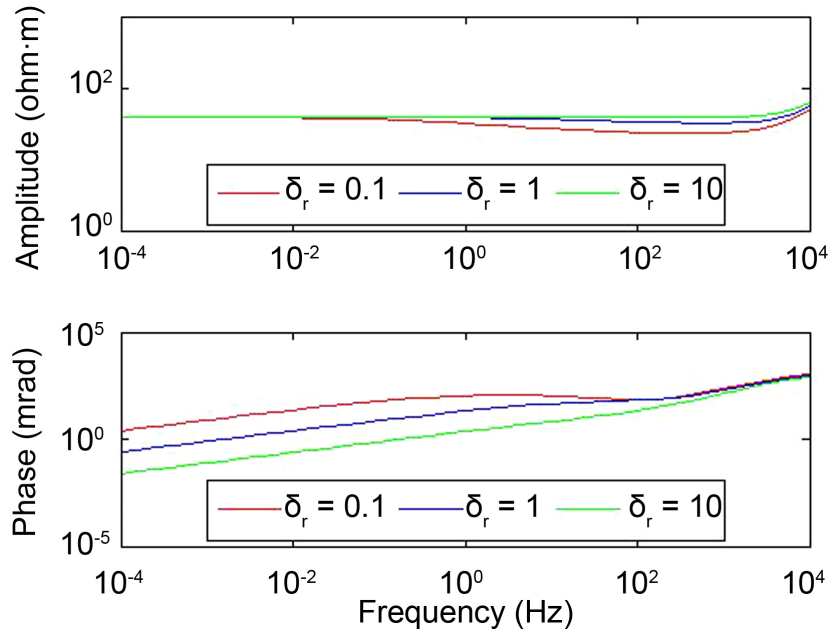

(c)

Figure 5. Amplitude and phase angle of the complex apparent resistivity in a well presenting mud, an invaded zone and formation. The invaded zone and formation are polarizable, and the intrinsic electrical properties are given by the fractal model when varying the parameter $\delta_{r}$. The radii of the invaded zone were (a) the same; (b) twice and (c) five times the well radius.
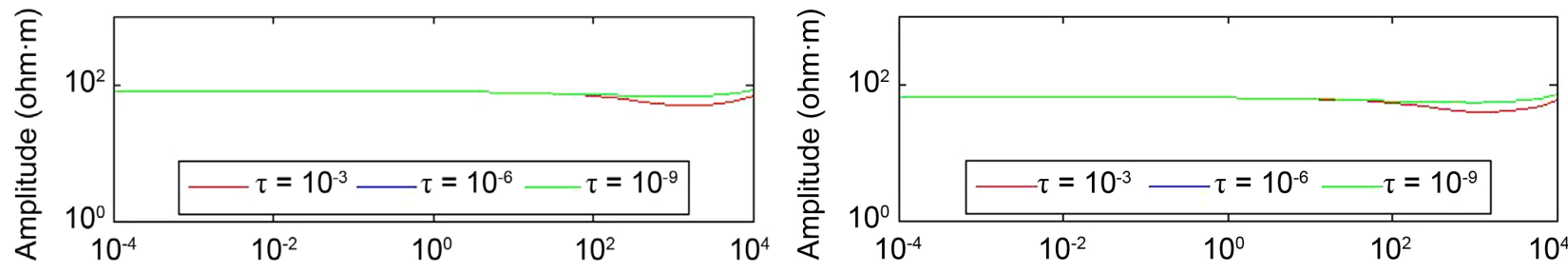

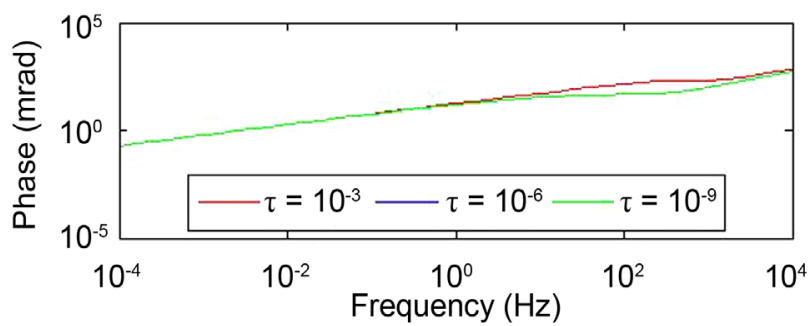

(a)

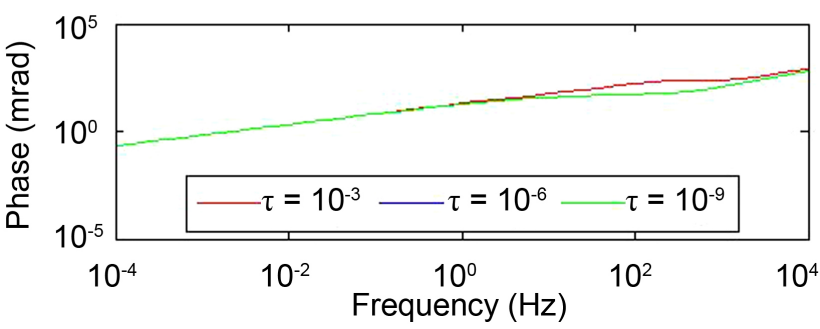

(b) 

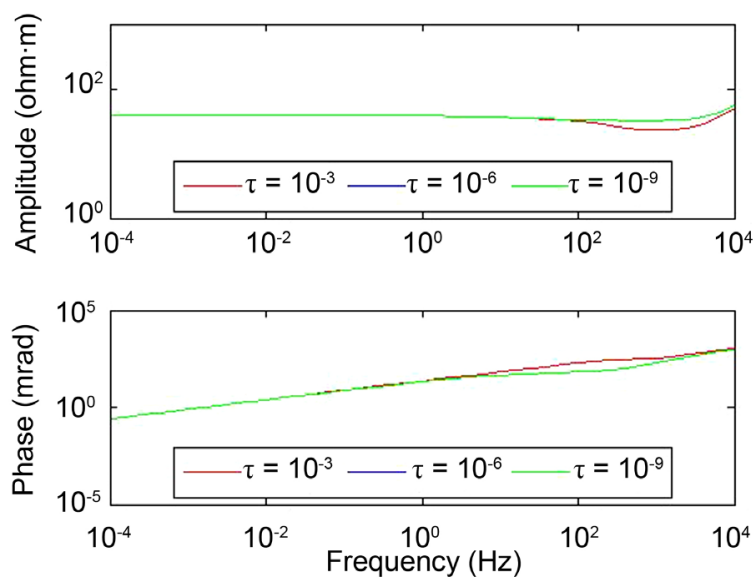

(c)

Figure 6. Amplitude and phase angle of the complex apparent resistivity in a well presenting mud, an invaded zone and formation. The invaded zone and formation are polarizable, and the intrinsic electrical properties are given by the fractal model when varying the time constant parameter $\tau$. The radii of the invaded zone were (a) the same; (b) twice and (c) five times the well radius.
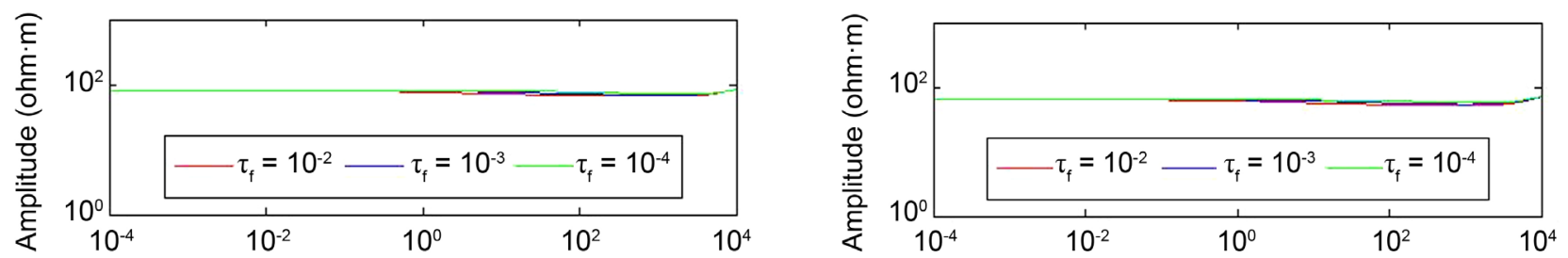

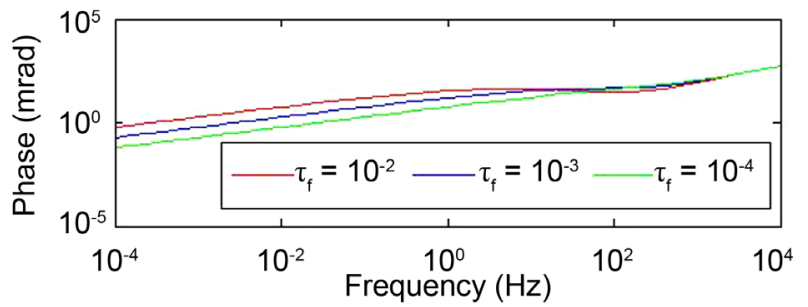

(a)

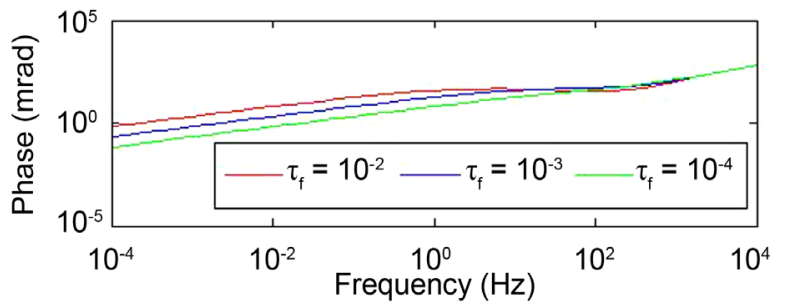

(b)
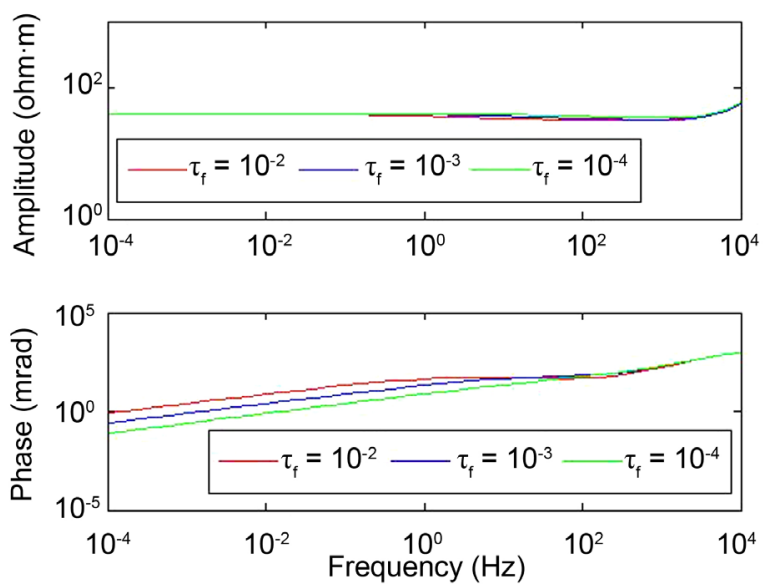

(c)

Figure 7. Amplitude and phase angle of the complex apparent resistivity in a well presenting mud, an invaded zone and formation. The invaded zone and formation are polarizable, and the intrinsic electrical properties are given by the fractal model when varying the fractal time constant parameter $\tau_{f}$ The radii of the invaded zone were (a) the same; (b) twice and (c) five times the well radius. 
investigated. The results demonstrate that, as in the case of an environment presenting horizontal layers, the parameters of the fractal model dominate the response phase of the complex apparent resistivity of the environment at low frequencies, with particular emphasis on the fractal exponent parameter $(\eta)$, the parameter relating the resistivity of the conductive grains that block the pores of the geological environment to the value of the DC resistivity of the rock matrix $\left(\delta_{r}\right)$ and the time parameter of fractal relaxation $\left(\tau_{f}\right)$. Since the induced polarization parameters at low frequencies carry information regarding the roughness of the rock pores, this result becomes important when interpreting petrophysical data. Furthermore, as the fractal exponent depends only on the mineralogical composition and the fractal geometry of the environment, the effects of the invaded zone in the phase response are attenuated. Additionally, the induced polarization data makes it possible to determine the characteristics of the environment without noticeable electromagnetic coupling effects at frequencies lower than $10^{4} \mathrm{~Hz}$.

\section{References}

[1] Da Rocha, B.R.P., Tejo, A.F., Valle, R.R.M. and Melo, M.A.B. (1997) Fractal Eletromagnetic Model for Biological Systems. Progress in Eletromagnetic Research, 1, 101-101.

[2] Da Rocha, B.R.P. and Habashy, T.M. (1995) Fractal Geometry, Porosity and Complex Resistivity I: From Rough Pore Interfaces to the Hand Specimens. In: Lovell, M.A., Ed., Developments in Petrophysics, London Geological Society, Special Publication, London, 277-286.

[3] Da Rocha, B.R.P. and Habashy, T.M. (1995) Fractal Geometry, Porosity and Complex Resistivity II: From Hand Specimens to Field Data. In: Lovell, M.A., Ed., Developments in Petrophysics, London Geological Society, Special Publication, London, 287-296.

[4] Sampaio, E.E.S., Santos, A.B. and Sato, H.K. (1998) Spectral Induced Polarization and Mineral Discrimination. Presented at the Society of Exploration of Geophysicists 68th Annual Meeting, Expanded Abstract.

[5] Kemna, A., Räkers, E. and Dresen, L. (1999) Field Application of Complex Resistivity Tomography. Presented at the Society of Exploration of Geophysicists 69th Annual Meeting, Expanded Abstract.

[6] Kemna, A., Binley, A., Ramírez, A. and Daily, W. (2000) Complex Resistivity Tomography for Environmental Applications. Chemical Engineering Journal, 77, 11 18. https://doi.org/10.1016/S1385-8947(99)00135-7

[7] Debye, P. (1929) Polar Molecules. The Chemical Catalog Co. Inc., New York.

[8] Cole, K.S. and Cole, R.H. (1941) Dispersion and Absorption in Dielectrics. The Journal of Chemical Physics, 9, 341-351. https://doi.org/10.1063/1.1750906

[9] Davidson, D.W. and Cole, R.H. (1951) Dielectric Relaxation in Glycerol, Propylene Glycol and n-Propano. The Journal of Chemical Physics, 19, 1484-1490. https://doi.org/10.1063/1.1748105

[10] Dias, C. A. (1972) Analytical Model for a Polarizable Medium at Radio and Lower Frequencies. Journal de Geophysical Research, 77, 4945-4956. https://doi.org/10.1029/JB077i026p04945

[11] Da Rocha, B.R.P. (1995) Modelo fractal para resistividade complexa de rochas: Sua 
interpretação petrofísica e aplicação à exploração geoelétrica. Tese de doutorado, Centro de Geociências, Universidade Federal do Pará.

[12] Farias, V.J.C., Maranhão, C.H.M., Rocha, B.R.P. and Andrade, N.P.O. (2010) Induced Polarization forward Modelling Using Finite Element Method and the Fractal Model. Applied Mathematical Modelling, 34, 1849-1860.

[13] Farias, V.J.C., Rocha, B.R.P., Rocha, M.P.C. and Tavares, H.R. (2013) The Use of the Fractal Model to Complex Resistivity in the Interpretation of Induced Polarization Data. Applied Mathematical Modelling, 37, 1347-1361.

[14] Seigel, H. (1959) Mathematical Formulation and Type Curves for Induced Polarization. Geophysics, 24, 547-563. https://doi.org/10.1190/1.1438625

[15] Gradshteyn, I.S. and Ryzhik (1980) Table of Integrals, Series, and Products. Academic Press, New York, 1160 p.

[16] Davis, P.J. and Rabinowitz, P. (1975) Methods of Numerical Integration. Academic Press, New York, 459 p.

[17] Rijo, L. (1992) An Optimized Digital Filter for the Cosine Transform. Revista Brasileira de Geofísica, 10, 7-20.

[18] Moran, J.H. and Gianzero, S. (1979) Effects of Formation Anisotropy on Resistivity-Logging Measurements. Geophysics, 44, 1266-1286.

https://doi.org/10.1190/1.1441006

[19] Freedman, R. and Vogiatzis, J.P. (1986) Theory of Induced-Polarization Logging in a Borehole. Geophysics, 51, 1830-1849. https://doi.org/10.1190/1.1442229

Submit or recommend next manuscript to SCIRP and we will provide best service for you:

Accepting pre-submission inquiries through Email, Facebook, LinkedIn, Twitter, etc. A wide selection of journals (inclusive of 9 subjects, more than 200 journals)

Providing 24-hour high-quality service

User-friendly online submission system

Fair and swift peer-review system

Efficient typesetting and proofreading procedure

Display of the result of downloads and visits, as well as the number of cited articles

Maximum dissemination of your research work

Submit your manuscript at: http://papersubmission.scirp.org/

Or contact ijg@scirp.org 